\title{
Genetic association study of PDLIM5 and HTR2A variants in Malaysian subjects diagnosed with bipolar disorder; a genetic modelling approach
}

\author{
Mohd Aizat Zain ${ }^{1, *}$, Nor Zuraida Zainal ${ }^{2}$, Sharmilla Kanagasundram ${ }^{2}$ and Zahurin Mohamed ${ }^{1}$ \\ ${ }^{1}$ Department of Pharmacology, Faculty of Medicine, University of Malaya, 50603 Kuala Lumpur, Wilayah \\ Persekutuan, Malaysia. \\ ${ }^{2}$ Department of Psychology Medicine, Faculty of Medicine, University of Malaya, 50603 Kuala Lumpur, Wilayah \\ Persekutuan, Malaysia.
}

*Correspondence: aizat03@gmail.com; Tel.: +603-79677896

Received: 30 April 2018; Accepted: 1 August 2018; Published: 8 August 2018

Edited by: King-Hwa Ling (Universiti Putra Malaysia, Malaysia)

Reviewed by: Azlina Ahmad Annuar (University of Malaya, Malaysia); Anonymous Reviewer 2 (Australia)

DOI: https://doi.org/10.31117/neuroscirn.v1i1.9

\begin{abstract}
Genetic hereditary has been implicated in bipolar disorder pathogenesis. The PDLIM5 and HTR2A genes have been investigated for its association with bipolar disorder in various populations, however, the results have been conflicting. In this study, we investigate the association between bipolar disorder and the two genes of interest, PDLIM5 and HTR2A genes. We recruited 253 bipolar disorder patients ( 75 Malays, 104 Chinese, and 74 Indians) and 505 control individuals (198 Malays, 155 Chinese, and 152 Indians) from three ethnic groups within Malaysian population. We genotyped for 3 SNPs of the PDLIM5 (rs2433320, rs2433322 and rs2438146) and 3 SNPs of the HTR2A (rs6313, rs2070040 and rs6311). Significant associations between bipolar disorder and each of the 3 SNPS of PDLIM5 in Malays, Indians and pooled samples. However, only rs2438146 remains significant in the Malays as co-dominant $(T / T$ vs. $C / C, p=0.004, O R=0.128$, $95 \% \mathrm{Cl}=0.031-0.524)$ and recessive genetic models ( $T / T$ vs. $C / T+C / C, p=0.003, O R=0.122$, $95 \% \mathrm{Cl}=0.030-0.494)$ after applying conservative Bonferroni correction. Haplotype analysis of 3 SNPS of PDLIM5 also showed a significant association with bipolar disorder. No association was observed between bipolar disorder and each of the 3 SNPS of HTR2A in any of the ethnicities. We conclude that PDLIM5 polymorphisms are associated with bipolar disorder in the pooled analysis. After stratification to different ethnic groups, the association remains significant in the Malay and Indian groups. The association is also supported by the significant association in haplotype analysis of PDLIM5. We also conclude there is no association between the HTR2A polymorphisms in the Malaysian population.
\end{abstract}

Keywords: PDLIM5; HTR2A; polymorphism; bipolar disorder 
C2018 by Zain et al for use and distribution in accord with the Creative Commons Attribution (CC BY-NC 4.0) license (https://creativecommons.org/licenses/by-nc/4.0/), which permits unrestricted non-commercial use, distribution, and reproduction in any medium, provided the original author and source are credited.

\section{Introduction}

Bipolar disorder (BPD), also known as manic-depressive illness, is a mental disorder characterized by typical symptoms of mania and depression which manifested as an elevated mood, hyperactive, unusual talkativeness, unusual thought patterns, extreme sadness or hopelessness and in severe cases, psychosis. The symptoms could be severe and may result in damaged relationships, career failure and even suicide. Family, twin and adoption studies suggest a strong genetic aetiology with an estimated genetic hereditary of $63 \%$ [1].

The PDLIM5 gene, or also known as PDZ and LIM domains, encodes the enigma homologue (ENH) which comprises of one PDZ domain located on the $\mathrm{N}$ terminal and 1-3 domains located on the C-terminal. The PDLIM5 lies on chromosome $4 \mathrm{q} 22$, found to be linked with BPD [2] ], major depression []ㅡ and schizophrenia $[4,5]$ through several linkage studies. The PDLIM5 gene is ubiquitously expressed in the brain such as in the hippocampus, thalamus, hypothalamus, cortex, and amygdala and its cellular localization is identical with Synapsin I, which is known to be involved in neurotransmitter release [6]. While the $P D Z$ domain of the PDZ-LIM protein has been demonstrated to be associated with the actin cytoskeleton [7]], the LIM domain of PDZ-LIM proteins is known to be associated with the kinase protein $[\underline{8}, \underline{9}]$. Furthermore, the LIM domain was also found able to regulate the protein kinase $C(P K C)$ activities in a PKC isoform-specific manner [10] and acts as an adaptor to PKC and N-type calcium channel []]. Abnormalities in PKC activity had been suggested to be involved in the pathophysiology of BPD [11].

Previous gene expression studies revealed dysregulation of PDLIM5 mRNA expression in the postmortem brain tissues of BPD patients. It was reported that the expression level of LIM mRNA was significantly increased in the post-mortem brain tissues BPD patient [12]. Interestingly, PKC activities have also been found to be increased in post-mortem brain tissues of patient with BPD [13]. Besides, PDLIM5 were found to be involved in the regulation of dendritic spine morphogenesis which was associated with BPD [14]. Recent information from animal behavioural study also showed that the PDLIM5 was implicated in the development of psychiatric symptoms, including mood disorder [15]. The involvement of PDLIM5 in mental disorders was supported by genetic association studies that relate several polymorphisms on PDLIM5 with mental disorders. For examples, the single nucleotide polymorphism (SNP) rs2433320 is associated with schizophrenia [16], major depression [17] and BPD [1ㅡ20]. Moreover, PDLIM5 SNP rs2433322 has been found associated with schizophrenia [17] whereas another SNP, rs2438146 is associated with BPD in its allelic frequencies but not genotype frequencies [18]. In addition, a previous study showed that rs 2433322 and rs2438146 did not associate with BPD in its own, but the haplotypes constructed from three SNPs of rs2433320-rs2438146-rs2433322 were significantly associated with BPD [20]. There were also several studies failed to replicate the significant association found in schizophrenia [21], major depression [22] and BPD [23].

A serotonin or 5-hydroxytryptamine $(5-\mathrm{HT})$, is a neurotransmitter in the brain that involved in many physiological roles including developmental, cardiovascular, gastrointestinal, and endocrine function, sensory perception, behaviours such as memory and learning, mood, sexual desires, sleep and cognition [24]. Serotonin-2A receptor (HTR2A) is a subtype of serotonin gene families and is located on chromosome 13q14-q21. The HTR2A has been associated with neuropsychiatric disorder like schizophrenia, BPD, obsessive compulsive disorder, anorexia and major depressive disorder, and 
cardiovascular disorders such as atherosclerosis and hypertension [25]. In gene expression study, the HTR2A mRNA was reported to be upregulated in the peripheral blood of major depression patients and correlated with severity and duration of depression illness [26]. In BPD particularly, studies based on postmortem brain, cerebrospinal fluid (CSF), neuroendocrine, genetic, platelet and psychopharmacological studies have shown that serotonin plays a pivotal role in the pathophysiology of BPD [26]. However, inconsistent results have been observed in previous genetic association studies; even though in general more studies failed to find a significant association between HTR2A polymorphism and bipolar disorder [27-29]. Inconsistency in the results reported for both genes of PDLIM5 and HTR2A in genetic association studies of BPD may be attributed to genetic heterogeneity in certain populations or methodological diversity among studies.

In the present study, we genotyped three SNPs of PDLIM5 (rs2433320, rs2433322 and rs2438146) and three SNPs of HTR2A (rs6313, rs2070040 and rs6311) which previously reported to have conflicting result in their association with BPD. We performed a casecontrol study involving Malaysian subjects diagnosed with BPD and analysed the data based on four different genetic models which are multiplicative (allelic), co-dominant, recessive and dominant models. Besides, we also performed haplotype analysis to identify the effects of multiple SNPs of PDLIM5 and HTR2A in BPD.

\section{Materials and Methods}

\subsection{Samples recruitment}

The study population consisted of 253 bipolar disorder patients (male $=131$, female $=122$, mean age $43.25 \pm 12.35$ years), and 505 control individuals (male $=298$, female=207, mean age $33.81 \pm 12.29$ ). The number of patients after stratification into ethnicities were 75 Malays (mean age $39.93 \pm 12.33$ ), 104 Chinese (mean age $43.47 \pm 12.35$ ) and 74 Indians (mean age 46.52 \pm 11.61 ) with bipolar disorder, whereas the number of control participants were 198 Malays (mean age 31.97 \pm 11.41 ), 155 Chinese (mean age

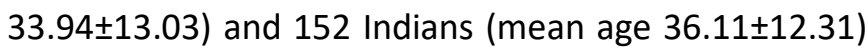
each.

Recruitment of study cases was carried out in the inpatient and outpatient psychiatric clinics of two medical centres, namely, the Universiti Malaya Medical Centre (UMMC), and the Universiti Kebangsaan Malaysia Medical Centre. All patients fulfilled the criteria of Diagnostic and Statistical Manual of Mental Disorders, $4^{\text {th }}$ ed. for bipolar disorder. Control individuals were recruited from among the staff and students of the University of Malaya and from the general medical clinics of UMMC who had been confirmed to be free from chronic diseases. The protocol used in this study was approved by the Medical Ethics Committee of UMMC, and this study was conducted in accordance with the Declaration of Helsinki. All the participants signed a consent form before participating in the study and an absence of inter-ethnic marriage for at least three previous generations was self-reported. These individuals were also checked for an absence of concurrent chronic diseases and/or alcoholism and substance abuse. Furthermore, all cases and controls were interviewed to obtain socio-demographic details including age, sex, marital status, ethnicity and occupation.

\subsection{SNPs selection and genotyping}

There were three SNPs of PDLIM5 (rs2433320, rs2433322 and rs2438146) and three SNPs of HTR2A (rs6313, rs2070040 and rs6311) which were genotyped using Real-Time PCR (RT-PCR). The SNPs information based on dbSNP database of National Centre for Biotechnology Information (NCBI) was assessed on 19 Jun 2018 [30] and was summarized in Table 1. Four genetic models were tested; multiplicative ( $A$ vs. $B$ ), codominant ( $A A$ vs. $B B ; A B$ vs. $B B$ ), recessive ( $A A$ vs. $B B+$ $A B)$ and dominant $(A A+A B$ vs. $B B)$ models $-A$ and $B$ are alleles for variant and wild-type, respectively. The DNA of the patients and control individuals were extracted from the blood samples using the QIAamp DNA Mini Kit (Qiagen, Hilden, Germany) and followed the standard protocols provided by the manufacturer. 
Table 1: Basic information of three polymorphisms of PDLIM5 and HTR2A genes

\begin{tabular}{|c|c|c|c|c|c|c|c|}
\hline Gene & SNPs & $\begin{array}{l}\text { Gene } \\
\text { position }\end{array}$ & Heterozygosity & MAF* & Functions & $\begin{array}{c}\text { mRNA } \\
\text { position }\end{array}$ & $\begin{array}{c}\text { Maj/Min } \\
\text { alleles }\end{array}$ \\
\hline \multirow[t]{3}{*}{ PDLIM5 } & Rs2433320 & Upstream & $N D^{a}$ & 0.272 & - & - & $\mathrm{G} / \mathrm{A}$ \\
\hline & Rs2438146 & Intron & 0.406 & 0.277 & - & - & $\mathrm{C} / \mathrm{T}$ \\
\hline & Rs2433322 & Intron & 0.406 & 0.272 & - & - & $A / G$ \\
\hline \multirow[t]{3}{*}{$H T R 2 A$} & Rs6313 & Intron & 0.486 & 0.441 & Synonymous & 791 & $\mathrm{G} / \mathrm{A}$ \\
\hline & Rs2070040 & Intron & 0.479 & 0.334 & - & - & $\mathrm{G} / \mathrm{A}$ \\
\hline & Rs6311 & Promoter & $N D^{a}$ & 0.444 & - & - & $\mathrm{C} / \mathrm{T}$ \\
\hline
\end{tabular}

${ }^{\mathrm{a}} \mathrm{ND}=$ not determined; $*$ MAF = Minor Allele Frequency; Maj/Min = Major/Minor

Genotyping by RT-PCR was performed by following the manufacturer's protocol and the probe of the SNPs used were commercially available (rs2433320, C_16015055_20; rs2438146, C_2095071_10; rs2433322, C_16015054_10; rs6313, C_3042197_1; rs2070040, C_16285134_10; rs6311, C_8695278_10) (Applied Biosystem, CA, USA).

\subsection{Statistical analysis}

The output of the genotype data was analysed using Microsoft Excel (Microsoft, Redmond, Washington, USA) and SPSS Statistical Software (SPSS Inc., Chicago, Illinois, USA). The Hardy-Weinberg equilibrium (HWE) values for both SNPs were calculated using calculator online tools (Court, 2005). The odds ratio (OR) with 95\% confidence interval $(\mathrm{Cl})$ were adjusted for age at study and sex through binary logistic regression analysis in all ethnic groups, while, the OR with $95 \% \mathrm{Cl}$ were adjusted for ethnicity, age at study and sex in the pooled population. Two-sided tests of statistical significance were used to determine statistically significant $p$-values $(p<0.05)$, followed by Bonferroni correction for multiple tests of the six SNPs in patients and controls ( $p=0.05 / 6$ SNPs; $p=0.0083$ ). To avoid increasing Type II error rate, the Bonferroni correction was corrected for only the number of SNPs as it was an actual variable tested in the present study. The power of this study was determined and calculated to estimate the sample size by using Power for Association with Error online tools (Rockefeller University, New York, New York, USA) [31]. Haplotype and linkage disequilibrium (LD) analysis for both SNPS of PDLIM5 was carried out using SHEsis and SHEsisPlus online tools (SHEsis; Bio-X Life Science Research Center, Shanghai, China) [32-34]. The p-value for haplotype analysis was adjusted according to Benjamini-Hochberg false-discovery rate (FDR) BH procedure.

\section{Results}

\subsection{BPD relationship with PDLIM5 and HTR2A SNPS}

Genotypic and allelic distributions of both PDLIM5 and HTR2A polymorphisms are summarized in Table 2 . The genetic models of both PDLIM5 and HTR2A polymorphisms in the Malay, Chinese, Indian and pooled population are presented in Table 3. The genotype distributions for all controls in both PDLIM5 and HTR2A polymorphisms were in Hardy-Weinberg equilibrium.

In the present study, we observed no association between the allelic frequencies of the six SNPs and BPD in every group (Table 3 ). In the Malays, there was a significant difference between case and control subjects in co-dominant and recessive models of rs2433320 (A/A vs. GG; A/A vs. G/A+G/G) and rs2438146 (T/T vs. $C / C ; T / T$ vs. $C / T+C / C$ ) and rs2433322 (A/G vs. $A / A ; G / G+A / G$ vs. $A / A)$ polymorphisms. While, no association was found between any PDLIM5 polymorphisms and BPD in the Chinese, the genotypes for co-dominant and recessive models of $r s 2438146$ (T/T vs. $C / C ; T / T$ vs. $C / T+C / C$ ) and rs2433322 (G/G vs. A/A; $G / G$ vs. $A / G+A / A)$ polymorphisms were significantly associated with BPD in the Indians. There was also a trend towards association in SNP rs2070040 under co-dominant (G/A 
vs. $\mathrm{G} / \mathrm{G} ; \mathrm{p}=0.06, \mathrm{OR}=0.712,95 \% \mathrm{Cl}=0.500-1.013$ ) model in the pooled subjects. After correction for multiple SNPs by using conservative Bonferroni correction, only SNP rs2438146 remains significant under co-dominant $(T / T$ vs. $C / C, p=0.004, O R=0.128$, $95 \% \mathrm{Cl}=0.031-0.524)$ and recessive $(T / T$ vs. $C / T+C / C$, $\mathrm{p}=0.003, \mathrm{OR}=0.122,95 \% \mathrm{Cl}=0.030-0.494)$ models in the Malays ( $p=0.0042$ after Bonferroni correction).

Table 2: Genotype and allele frequencies of PDLIM5 and HTR2A polymorphisms of case and control in three ethnic groups of the Malaysian population

\begin{tabular}{|c|c|c|c|c|c|c|c|c|}
\hline \multirow{2}{*}{$\begin{array}{l}\text { *Allele/ } \\
\text { Genotype }\end{array}$} & \multicolumn{2}{|c|}{ Malay } & \multicolumn{2}{|c|}{ Chinese } & \multicolumn{2}{|c|}{ Indian } & \multicolumn{2}{|c|}{ Pooled } \\
\hline & Case (\%) & Control (\%) & Case (\%) & Control (\%) & Case (\%) & Control (\%) & Case (\%) & Control (\%) \\
\hline \multicolumn{9}{|l|}{ PDLIM5 } \\
\hline \multicolumn{9}{|l|}{ rs2433320 } \\
\hline G & 155 (77) & $327(83)$ & $167(80)$ & 246 (79) & $116(80)$ & $245(81)$ & $398(79)$ & $818(81)$ \\
\hline$A$ & $35(23)$ & $69(17)$ & $41(20)$ & $64(21)$ & $30(20)$ & 57 (19) & $106(21)$ & 190 (19) \\
\hline GG & $47(63)$ & $132(67)$ & $67(64)$ & $100(64)$ & $47(64)$ & $100(66)$ & $161(64)$ & $332(66)$ \\
\hline$A G$ & $21(28)$ & $63(32)$ & $33(32)$ & $46(30)$ & $22(30)$ & $45(30)$ & $76(30)$ & $154(31)$ \\
\hline $\mathrm{AA}$ & 7 (9) & $3(1)$ & $4(4)$ & $9(6)$ & $4(6)$ & $6(4)$ & $15(6)$ & $18(3)$ \\
\hline \multicolumn{9}{|l|}{ rs2438146 } \\
\hline $\mathrm{C}$ & $113(75)$ & $327(83)$ & $174(84)$ & $246(79)$ & $107(72)$ & $245(81)$ & $394(78)$ & $818(81)$ \\
\hline $\mathrm{T}$ & $37(25)$ & $69(17)$ & $32(16)$ & $64(21)$ & $41(28)$ & 57 (19) & $110(22)$ & 190 (19) \\
\hline CC & 47 (63) & 132 (67) & $74(72)$ & $101(65)$ & $43(58)$ & $100(66)$ & $164(65)$ & $333(66)$ \\
\hline CT & $19(25)$ & $63(32)$ & $26(25)$ & $44(28)$ & $21(28)$ & $45(30)$ & $66(26)$ & $152(30)$ \\
\hline $\mathrm{TT}$ & $9(12)$ & $3(1)$ & $3(3)$ & $10(7)$ & $10(14)$ & $6(4)$ & $22(9)$ & $19(4)$ \\
\hline \multicolumn{9}{|l|}{ rs2433322 } \\
\hline A & $115(77)$ & $327(83)$ & $170(82)$ & $240(77)$ & $106(72)$ & $245(81)$ & $391(77)$ & $812(81)$ \\
\hline G & $35(23)$ & $69(17)$ & $38(18)$ & $70(23)$ & $42(28)$ & 57 (19) & $115(23)$ & 196 (19) \\
\hline$A A$ & $48(64)$ & 132 (67) & 70 (67) & $96(62)$ & $42(57)$ & $100(66)$ & $160(63)$ & $328(65)$ \\
\hline$A G$ & $19(25)$ & $63(32)$ & $30(29)$ & $48(31)$ & $22(30)$ & $45(30)$ & $71(28)$ & $156(31)$ \\
\hline GG & $8(11)$ & $3(1)$ & $4(4)$ & $11(7)$ & $10(14)$ & $6(4)$ & $22(9)$ & $20(4)$ \\
\hline \multicolumn{9}{|l|}{ HTR2A } \\
\hline \multicolumn{9}{|l|}{ rs6313 } \\
\hline G & $53(35)$ & $146(37)$ & $71(34)$ & $122(39)$ & $77(52)$ & $153(51)$ & $201(40)$ & $421(42)$ \\
\hline$A$ & $97(65)$ & $250(63)$ & $137(66)$ & $188(61)$ & $71(48)$ & 145 (49) & $305(60)$ & $583(58)$ \\
\hline GG & $11(15)$ & $35(18)$ & $11(11)$ & $21(13)$ & $19(26)$ & $43(29)$ & $41(16)$ & $99(20)$ \\
\hline GA & $31(41)$ & $76(38)$ & 49 (47) & $80(52)$ & $39(52)$ & $67(45)$ & $119(47)$ & $223(44)$ \\
\hline $\mathrm{AA}$ & $33(44)$ & $87(44)$ & $44(42)$ & $54(35)$ & $16(22)$ & $39(26)$ & $93(37)$ & $180(36)$ \\
\hline \multicolumn{9}{|l|}{ rs2070040 } \\
\hline G & 104 (69) & $286(72)$ & $138(66)$ & $215(70)$ & $97(66)$ & 202 (67) & $167(33)$ & $303(30)$ \\
\hline$A$ & $46(31)$ & $110(28)$ & $70(34)$ & $93(30)$ & $51(34)$ & $100(33)$ & $339(67)$ & 703 (70) \\
\hline GG & 37 (49) & $111(56)$ & $43(41)$ & 73 (47) & $30(40)$ & $68(45)$ & $110(44)$ & $252(50)$ \\
\hline$A G$ & $30(40)$ & $64(32)$ & $52(50)$ & $69(45)$ & $37(50)$ & $66(44)$ & 119 (47) & $199(40)$ \\
\hline $\mathrm{AA}$ & $8(11)$ & $23(12)$ & $9(9)$ & $12(8)$ & 7 (10) & $17(11)$ & $24(10)$ & $52(10)$ \\
\hline \multicolumn{9}{|l|}{ rs6311 } \\
\hline $\mathrm{C}$ & $52(35)$ & $143(36)$ & 73 (35) & $120(40)$ & $76(52)$ & 146 (49) & $201(40)$ & 409 (41) \\
\hline $\mathrm{T}$ & $96(65)$ & $253(64)$ & $135(65)$ & $184(60)$ & $70(48)$ & $154(51)$ & $301(60)$ & $591(59)$ \\
\hline CC & $12(16)$ & $33(17)$ & $11(11)$ & $21(14)$ & $19(26)$ & $37(25)$ & 42 (17) & $91(18)$ \\
\hline CT & 28 (39) & 77 (39) & 51 (49) & $78(52)$ & $38(52)$ & $72(48)$ & $117(47)$ & $227(46)$ \\
\hline TT & $34(46)$ & $88(44)$ & $42(40)$ & $53(35)$ & $16(22)$ & $41(27)$ & $92(37)$ & $182(36)$ \\
\hline
\end{tabular}

\footnotetext{
* Few samples failed to be genotyped which resulted a slightly difference in the number of sample and number of genotypes.
} 
Table 3: Genotype and allelic frequencies of PDLIM5 and HTR2A polymorphisms in case and control subjects in total population and in the three ethnic subgroups under alternative genetic models.

\begin{tabular}{|c|c|c|c|c|c|c|c|c|c|c|c|c|c|c|c|c|}
\hline \multirow{3}{*}{ Genotype } & \multicolumn{12}{|c|}{ Ethnicity } & \multirow{2}{*}{\multicolumn{4}{|c|}{ Pooled }} \\
\hline & \multicolumn{4}{|c|}{ Malay } & \multicolumn{4}{|c|}{ Chinese } & \multicolumn{4}{|c|}{ Indian } & & & & \\
\hline & Cs & Ctl & $*_{p}$ & ${ }^{\mathrm{a}} \mathrm{OR}(95 \% \mathrm{Cl})$ & Cs & CtI & $* p$ & ${ }^{\mathrm{a}}$ OR (95\% Cl) & Cs & CtI & $*_{p}$ & ${ }^{\mathrm{a}} \mathrm{OR}(95 \% \mathrm{Cl})$ & Cs & CtI & $*_{p}$ & ${ }^{\mathrm{a}} \mathrm{OR}(95 \% \mathrm{Cl})$ \\
\hline \multicolumn{17}{|l|}{ rs2433320 } \\
\hline A vs. $G$ & 35 & 69 & 0.19 & $0.168(0.038-0.744)$ & 41 & 64 & 0.30 & $2.390(0.463-12.326)$ & 30 & 57 & 0.34 & $0.460(0.099-2.144)$ & 106 & 190 & 0.24 & $0.615(0.272-1.390)$ \\
\hline G/A vs. G/G & 47 & 132 & 0.83 & $1.067(0.588-1.939)$ & 33 & 46 & 0.56 & $0.835(0.452-1.541)$ & 22 & 45 & 0.78 & $1.107(0.548-2.223)$ & 76 & 154 & 0.74 & $0.939(0.651-1.356)$ \\
\hline A/A vs. G/G & 7 & 3 & 0.01 & $0.155(0.038-0.624)$ & 4 & 9 & 0.34 & $2.256(0.432-11.783)$ & 4 & 6 & 0.35 & $0.475(0.100-2.245)$ & 15 & 18 & 0.23 & $0.603(0.264-1.375)$ \\
\hline$A / A$ vs. $G / A+G / G$ & 7 & 3 & 0.02 & $0.168(0.038-0.744)$ & 4 & 9 & 0.30 & $2.390(0.463-12.326)$ & 4 & 6 & 0.32 & $0.460(0.099-2.144)$ & 15 & 18 & 0.24 & $0.615(0.272-1.390)$ \\
\hline $\begin{array}{l}\mathrm{G} / \mathrm{A}+\mathrm{A} / \mathrm{A} \text { vs. } \mathrm{G} / \mathrm{G} \\
\text { rs2438146 }\end{array}$ & 28 & 65 & 0.43 & $0.786(0.435-1.420)$ & 37 & 55 & 0.81 & $0.928(0.514-1.675)$ & 26 & 51 & 1.00 & $1.002(0.514-1.954)$ & 91 & 172 & 0.52 & $0.890(0.627-1.264)$ \\
\hline T vs. C & 37 & 69 & 0.53 & $0.827(0.458-1.490)$ & 32 & 64 & 0.26 & $1.415(0.772-2.592)$ & 41 & 57 & 0.48 & $0.788(0.410-1.514)$ & 110 & 190 & 0.88 & $0.973(0.685-1.381)$ \\
\hline $\mathrm{C} / \mathrm{T}$ vs. $\mathrm{C} / \mathrm{C}$ & 19 & 63 & 0.65 & $1.161(0.606-2.224)$ & 26 & 44 & 0.45 & $1.281(0.675-2.429)$ & 21 & 45 & 0.79 & $1.104(0.536-2.273)$ & 66 & 152 & 0.01 & $2.664(1.261-5.627)$ \\
\hline $\mathrm{T} / \mathrm{T}$ vs. $\mathrm{C} / \mathrm{C}$ & 9 & 3 & $<0.01$ & $0.128(0.031-0.524)$ & 3 & 10 & 0.21 & $2.474(0.603-10.151)$ & 10 & 6 & 0.01 & $0.191(0.054-0.677)$ & 22 & 19 & 0.02 & $2.310(1.139-4.683)$ \\
\hline$T / T$ vs. $C / T+C / C$ & 9 & 3 & $<0.01$ & $0.122(0.030-0.494)$ & 3 & 10 & 0.24 & $2.308(0.569-9.363)$ & 10 & 6 & 0.01 & $0.185(0.053-0.645)$ & 22 & 19 & 0.01 & $2.410(1.199-4.844)$ \\
\hline$T / T+C / T$ vs. $C / C$ & 28 & 66 & 0.53 & $0.827(0.458-1.490)$ & 29 & 54 & 0.26 & $1.415(0.772-2.592)$ & 31 & 51 & 0.48 & $0.788(0.410-1.514)$ & 88 & 171 & 0.88 & $1.028(0.724-1.459)$ \\
\hline \multicolumn{17}{|l|}{ rs2433322 } \\
\hline G vs. A & 35 & 69 & 0.54 & $0.829(0.458-1.502)$ & 38 & 70 & 0.46 & $1.251(0.695-2.254)$ & 42 & 57 & 0.73 & $3.727(0.379-1.394)$ & 115 & 196 & 0.65 & $0.923(0.652-1.306)$ \\
\hline$A / G$ vs. $A / A$ & 19 & 63 & 0.01 & $0.144(0.034-0.606)$ & 30 & 48 & 0.17 & $2.490(0.673-9.212)$ & 22 & 10 & 0.99 & $0.993(0.486-2.030)$ & 71 & 156 & 0.79 & $1.052(0.724-1.528)$ \\
\hline G/G vs. A/A & 8 & 3 & 0.74 & $1.116(0.583-2.137)$ & 4 & 11 & 0.77 & $1.097(0.588-2.047)$ & 10 & 6 & 0.01 & $0.185(0.052-0.658)$ & 22 & 328 & 0.04 & $0.485(0.240-0.981)$ \\
\hline$G / G$ vs. $A / G+A / A$ & 8 & 3 & 0.01 & $0.139(0.033-0.579)$ & 4 & 11 & 0.18 & $2.421(0.663-8.834)$ & 10 & 6 & 0.01 & $0.185(0.053-0.645)$ & 22 & 328 & 0.04 & $0.478(0.238-0.957)$ \\
\hline $\begin{array}{l}G / G+A / G \text { vs. } A / A \\
\text { rs6313 }\end{array}$ & \multicolumn{16}{|c|}{ rs6313 } \\
\hline A vs. G & 97 & 250 & 0.15 & $0.553(0.246-1.247)$ & 137 & 188 & 0.41 & $0.690(0.283-1.678)$ & 71 & 145 & 0.86 & $0.936(0.458-1.916)$ & 305 & 583 & 0.10 & $0.691(0.444-1.077)$ \\
\hline G/A vs. G/G & 31 & 76 & 0.20 & $0.572(0.241-1.356)$ & 49 & 80 & 0.21 & $0.541(0.206-1.422)$ & 39 & 67 & 0.50 & $0.769(0.361-1.639)$ & 119 & 223 & 0.11 & $0.678(0.424-1.086)$ \\
\hline A/A vs. G/G & 33 & 87 & 0.16 & $0.533(0.222-1.279)$ & 44 & 54 & 0.65 & $0.806(0.320-2.032)$ & 16 & 39 & 0.44 & $1.442(0.573-3.631)$ & 93 & 180 & 0.17 & $0.709(0.433-1.162)$ \\
\hline$A / A$ vs. $G / A+G / G$ & 33 & 87 & 0.70 & $0.895(0.506-1.584)$ & 44 & 54 & 0.15 & $0.645(0.356-1.170)$ & 16 & 39 & 0.18 & $1.712(0.786-3.726)$ & 93 & 180 & 0.72 & $0.923(0.659-1.335)$ \\
\hline $\begin{array}{l}\text { A/A+G/A vs. } G / G \\
\text { rs2070040 }\end{array}$ & 64 & 163 & 0.15 & $0.553(0.246-1.247)$ & 93 & 134 & 0.41 & $0.69(0.283-1.678)$ & 55 & 106 & 0.86 & $0.936(0.458-1.916)$ & 212 & 403 & 0.10 & $0.691(0.444-1.077)$ \\
\hline A vs. G & 46 & 110 & 0.40 & $1.497(0.582-3.847)$ & 70 & 93 & 0.94 & $1.043(0.371-2.937)$ & 51 & 100 & 0.69 & $1.312(0.427-4.032)$ & 339 & 703 & 0.33 & $1.331(0.746-2.372)$ \\
\hline G/A vs. G/G & 30 & 64 & 0.24 & $0.695(0.379-1.276)$ & 52 & 69 & 0.25 & $0.708(0.392-1.281)$ & 37 & 66 & 0.52 & $0.803(0.413-1.561)$ & 119 & 199 & 0.06 & $0.712(0.500-1.013)$ \\
\hline A/A vs. $G / G$ & 8 & 23 & 0.61 & $1.293(0.486-3.438)$ & 9 & 12 & 0.81 & $0.875(0.297-2.579)$ & 7 & 17 & 0.80 & $1.165(0.358-3.793)$ & 110 & 252 & 0.69 & $1.131(0.618-2.068)$ \\
\hline$A / A$ vs. $G / A+G / G$ & 8 & 23 & 0.40 & $1.497(0.582-3.847)$ & 9 & 12 & 0.94 & $1.043(0.371-2.937)$ & 7 & 17 & 0.64 & $1.312(0.427-4.032)$ & 110 & 252 & 0.33 & $1.331(0.746-2.372)$ \\
\hline $\begin{array}{l}A / A+G / A \text { vs. } G / G \\
\text { rs6311 }\end{array}$ & 38 & 87 & 0.45 & $0.803(0.455-1.414)$ & 61 & 81 & 0.28 & $0.731(0.413-1.294)$ & 44 & 83 & 0.62 & $0.851(0.448-1.618)$ & 229 & 451 & 0.14 & $0.775(0.553-1.084)$ \\
\hline T vs. C & 96 & 253 & 0.61 & $0.860(0.485-1.525)$ & 135 & 184 & 0.49 & $0.813(0.451-1.465)$ & 70 & 154 & 0.20 & $1.649(0.766-3.550)$ & 301 & 591 & 0.95 & $0.989(0.695-1.407)$ \\
\hline $\mathrm{C} / \mathrm{T}$ vs. $\mathrm{C} / \mathrm{C}$ & 28 & 77 & 0.34 & $0.654(0.273-1.563)$ & 51 & 78 & 0.52 & $0.737(0.295-1.845)$ & 38 & 72 & 0.75 & $1.137(0.524-2.464)$ & 117 & 227 & 0.37 & $0.805(0.501-1.291)$ \\
\hline$T / T$ vs. $C / C$ & 34 & 88 & 0.30 & $0.640(0.275-1.490)$ & 42 & 53 & 0.35 & $0.634(0.244-1.649)$ & 16 & 41 & 0.22 & $1.801(0.708-4.578)$ & 92 & 182 & 0.50 & $0.845(0.515-1.385)$ \\
\hline $\mathrm{T} / \mathrm{T}$ vs. $\mathrm{C} / \mathrm{T}+\mathrm{C} / \mathrm{C}$ & 34 & 88 & 0.61 & $0.860(0.485-1.525)$ & 42 & 53 & 0.49 & $0.813(0.451-1.465)$ & 16 & 41 & 0.20 & $1.649(0.766-3.550)$ & 92 & 182 & 0.95 & $0.989(0.695-1.407)$ \\
\hline$T / T+C / T$ vs. $C / C$ & 62 & 165 & 0.28 & $0.646(0.291-1.436)$ & 93 & 131 & 0.42 & $0.694(0.287-1.679)$ & 54 & 113 & 0.47 & $1.311(0.627-2.739)$ & 209 & 409 & 0.39 & $0.821(0.526-1.282)$ \\
\hline
\end{tabular}




\section{Continued from previous page}

Cs, Case: Ctl, Control.

a estimated odds ratio by binary logistic regression after adjustment to age at study, gender and ethnicity in pooled population, while the odds ratio was adjusted to age at study and gender in each ethnicity.

* p-value was rounded to two decimals point. Only SNP rs2438146 in Malay remains significant (bold) after Bonferroni correction was applied $p<0.0083$.

\subsection{Linkage disequilibrium (LD) and haplotype analysis}

Pairwise LD coefficients $r^{2}$ between PDLIM5 SNPs rs2433320-rs2438146-rs2433322 in the pooled population was less than 0.90. The LD coefficients $r^{2}$ at 0.90 and above was set as in LD. The LD for rs 2438146 and rs2433322 was at $r^{2}=0.86$. Moreover, the $r^{2}$ between HTR2A SNPs rs6313, rs2070040 and rs6311 in the pooled population did not reach 0.90 .

For haplotype analysis, we analysed only the common haplotypes (frequency $>0.03$ ) in the pooled population. We summarized haplotype analysis results in Table 4 and Table 5, with the column for case and control frequencies in both tables that were presented in percentage unit with zero decimal. In Table 4, we showed that G-C-A haplotype constructed from PDLIM5 SNPs rs2433320, rs2438146 and rs2433322 was the most frequent SNPs in case (0.74) and control (0.72) subjects. However, only A-C-A showed significantly associated after adjusted with FDR correction. The ratio of this wild-type haplotype (G-CA) to the mutant haplotype (A-C-A) frequency was 24.67 in case subject. While, in table 5, there was no association observed in any haplotype constructed between HTR2A SNPs rs6313, rs2070040 and rs6311.

Table 4: Haplotype frequencies of rs2433320, rs 2438146 and rs2433322 polymorphisms of PDLIM5 gene within case and control subjects in the pooled population.

\begin{tabular}{lccrc}
\hline \#Haplotypes & \multicolumn{4}{c}{ Pooled Population } \\
\cline { 2 - 5 } & Case (Freq \%) & Control (Freq \%) & p-value (*FDR q-value) & OR (Cl 95\%) \\
\hline G-C-A & $370(0.74)$ & $712(0.72)$ & $0.285(0.326)$ & $1.138(0.896-1.445)$ \\
A-T-G & $82(0.16)$ & $150(0.15)$ & $0.489(0.489)$ & $1.108(0.826-1.486)$ \\
A-C-A & $17(0.03)$ & $74(0.08)$ & $\mathbf{0 . 0 0 2 ( 0 . 0 0 3 )}$ & $0.439(0.256-0.753)$ \\
G-T-G & $22(0.04)$ & $28(0.03)$ & $0.105(0.14)$ & $1.594(0.902-2.815)$ \\
\hline
\end{tabular}

* The FDR q-value $<0.05$ is significant (bold). " Haplotypes with frequency $<0.03$ are ignored.

Table 5: Haplotype frequencies of rs6313, rs2070040 and rs6311 polymorphisms of HTR2A gene within case and control subjects in the pooled population.

\begin{tabular}{lcccc}
\hline \#Haplotypes & \multicolumn{4}{c}{ Pooled Population } \\
\cline { 2 - 5 } & Case (Freq \%) & Control (Freq \%) & p-value (*FDR q-value) & OR (CI 95\%) \\
\hline A-G-C & $277(0.551)$ & $531(0.534)$ & $0.424(0.543)$ & $1.091(0.88-1.351)$ \\
G-A-T & $143(0.284)$ & $268(0.269)$ & $0.475(0.543)$ & $1.09(0.859-1.384)$ \\
G-G-T & $53(0.105)$ & $98(0.098)$ & $0.636(0.636)$ & $1.088(0.765-1.549)$ \\
\hline
\end{tabular}

* The FDR q-value $<0.05$ is significant. ${ }^{*}$ Haplotypes with frequency $<0.03$ are ignored.

\section{Discussion}

The present study found a significant association between PDLIM5 SNPs (rs2433320, rs2438146 and rs2433322) and BPD in Malays, Indians and pooled Malaysian subjects. However, after performing conservative Bonferroni correction, only rs2438146 remains associated with BPD in the Malays. In addition, the haplotype analysis that we performed also supported the result of association found between
PDLIM5 and BPD in the Malays. While we observed a significant association between PDLIM5 and BPD, there was no association observed between HTR2A and BPD in any groups of the population tested in single SNP association and haplotype studies.

Previous genetic association studies of PDLIM5 SNP rs2433320 showed significant association in BPD [18], schizophrenia [16] and major depression [1]. 
However, there was a study that reported an absence of association between rs2433320 and BPD in the Han Chinese population [20], which is comparable with our result found in the present study with the Chinese group. The A allele of rs 2433320 has been suggested to associate with higher expression of PDLIM5 in the postmortem brain of schizophrenia [16], while in another study, the $G$ allele of rs2433320 has been suggested to have a protective role in major depression [17]. In the present study, the co-dominant model of rs2433320 was significantly increased in BPD of the Malays group, however, did not survive after strict Bonferroni correction. Our results suggest that the co-dominant model of rs2433320 may have a small effect size to show a true association.

Kato et al. (2005) and Zhao et al. (2009) reported significant associations between two SNPs of PDLIM5; rs2438146 and rs2433322, with BPD in Japanese (independent sample sets) and Han Chinese, respectively $[\underline{18}, 20]$. Zhao et al. (2009) [20] suggested that the $G$ allele of rs2433322 might be a risk factor in BPD as the allele appears to be in higher frequency in cases compared to control subjects. While, our result in Chinese did not support a significant association of rs2433322 and BPD [20], we did find a significant association in recessive model of rs2433322 and BPD in Malays and Indians. We also observed that the $G$ allele of rs2433322 appeared more in the case than in control subjects and thus supported previous suggestions that the $\mathrm{G}$ allele is a risk factor for BPD. The risk, however, is likely to be small $(O R<1)$. In addition, we also observed the co-dominant ( $T / T$ vs. CC) and recessive ( $T / T$ vs. $C / T+C / C)$ models of SNP rs2438146 remains significant even after Bonferroni correction. Our results may explain previous results which found no association observed in allelic (T vs. C) and genotype (T/T vs. T/C vs. C/C) frequencies between SNP rs2438146 and BPD; the discrepancies between the present and the previous study suggested that allele T of SNP rs2438146 carries more effect in the homozygous form of $T / T$, and loses its effect in the heterozygous form of $\mathrm{C} / \mathrm{T}$ as no significant observed under dominant $(T / T+C / T$ vs. $C / C)$ and co-dominant (C/T vs. C/C) models.

In the haplotype analysis, after corrected for FDR, we observed a significant association between A-C-A haplotype constructed from PDLIM5 SNPs rs2433320, rs2438146 and rs2433322, and BPD. The odds ratio produced was 0.439 at $95 \%$ confidence interval of 0.256-0.753, indicates protective nature of the haplotype.
In the present study, there are several limitations that need to be taken into consideration. Firstly, the sample size in this study after stratification into ethnicities did not achieve $80 \%$ of power of study. For example, for the SNP rs2438146 to achieve $80 \%$ power of study, it required a minimal sample size of 182 cases and 480 controls for Malay; 559 cases and 833 controls for Chinese; and 203 cases and 426 controls for Indian. Thus, we cannot exclude a false positive error from the analysis for each ethnic group. However, it must be noted that the present results are not conflicting with previous studies. Secondly, the SNPs rs2438146 and rs2433322 are in the deviation of HWE in the Malays and Indians cases (but not in controls). However, we did not find any genotyping errors via manual checks as suggested by Lewis (2002) [35]. It was explained that the cases would not be in HWE for a SNP with a true genetic effect that is not controlled by a multiplicative model. However, if the cases were in HWE, the data can be analysed by allele counting (multiplicative model) $[\underline{35}, \underline{36}]$.

\section{Conclusions}

In conclusion, we showed a significant association between polymorphisms of the PDLIM5 and BPD in Malays, Indians and pooled subjects. However, only the SNP rs2438146 remains significant in Malays after performing the correction for multiple tests. The significant association was strengthened by results of haplotype analysis of the PDLIM5 polymorphism. We did not detect any significant association between the screened SNPs or haplotypes of the HTR2A with BPD.

Acknowledgements: The author would like to thank all patients and staff from the University of Malaya Medical Centre and the National University of Malaysia Medical Centre who participated in this study. Special thanks to Dr. BS Haerian, Dr. SM Zain and Dr. Y Apalasamy and our colleagues in the PGX laboratory for contributing whether directly or indirectly in this study. This study was supported by University of Malaya Research Grant RG162/09HTM and RG184/10HTM, Postgraduate Research Grant of the University Malaya PS226/09HTM, and HIR MOHE Grant E00025-2001. 
Author Contributions: MAZ and ZM were involved in the study design, while MAZ carried out sample collection, performed genotyping and statistical analysis and drafted the manuscript. NZZ and SK were involved in the diagnosis of patients, provided clinical advice and were involved in applying for ethics permissions and grant funding, and helping in case subjects collection. ZM critically reviewed the manuscript.

Conflicts of Interest: The authors declare no conflict of interest.

\section{References}

1. Smoller J.W., Finn C.T. Family, twin, and adoption studies of bipolar disorder. Am J Med Genet C Semin Med Genet. 2003, 123C, 48-58. https://doi.org/10.1002/ajmg.c.20013

2. Detera-Wadleigh S.D., Badner J.A., Yoshikawa T., Sanders A.R., Goldin L.R., Turner G., et al. Initial genome scan of the NIMH genetics initiative bipolar pedigrees: chromosomes 4, 7, 9, 18, 19, 20, and 21q. Am J Med Genet. 1997, 74, 254262. https://doi.org/10.1002/(SICI)1096-8628(19970531)74:3\%3C254::AID-AJMG4\%3E3.0.CO;2-Q

3. Camp N.J., Lowry M.R., Richards R.L., Plenk A.M., Carter C., Hensel C.H., et al. Genome-wide linkage analyses of extended Utah pedigrees identifies loci that influence recurrent, early-onset major depression and anxiety disorders. Am J Med Genet B Neuropsychiatr Genet. 2005, 135B, 85-93. https://doi.org/10.1002/ajmg.b.30177

4. Kato T. Molecular genetics of bipolar disorder and depression. Psychiatry Clin Neurosci. 2007, 61, 3-19. https://doi.org/10.1111/j.1440-1819.2007.01604.x

5. Mowry B.J., Ewen K.R., Nancarrow D.J., Lennon D.P., Nertney D.A., Jones H.L., et al. Second stage of a genome scan of schizophrenia: study of five positive regions in an expanded sample. Am J Med Genet. 2000, 96, 864-869. https://doi.org/10.1002/1096-8628(20001204)96:6\%3C864::AID-AJMG35\%3E3.0.CO;2-D

6. Maeno-Hikichi Y., Chang S., Matsumura K., Lai M., Lin H., Nakagawa N., et al. A PKC epsilon-ENH-channel complex specifically modulates N-type Ca2+ channels. Nat Neurosci. 2003, 6, 468-475. https://doi.org/10.1038/nn1041

7. Vallenius T., Luukko K., Makela T.P. CLP-36 PDZ-LIM protein associates with nonmuscle alpha-actinin-1 and alphaactinin-4. J Biol Chem. 2000, 275, 11100-11105. https://doi.org/10.1074/jbc.275.15.11100

8. Kuroda S., Tokunaga C., Kiyohara Y., Higuchi O., Konishi H., Mizuno K., et al. Protein-protein interaction of zinc finger LIM domains with protein kinase C. J Biol Chem. 1996, 271, 31029-31032. https://doi.org/10.1074/ibc.271.49.31029

9. Zhou Q., Ruiz-Lozano P., Martone M.E., Chen J. Cypher, a striated muscle-restricted PDZ and LIM domain-containing protein, binds to alpha-actinin-2 and protein kinase C. J Biol Chem. 1999, 274, 19807-19813. https://doi.org/10.1074/ibc.274.28.19807

10. Maturana A.D., Nakagawa N., Yoshimoto N., Tatematsu K., Hoshijima M., Tanizawa K., et al. LIM domains regulate protein kinase $C$ activity: a novel molecular function. Cell Signal. 2011, 23, 928-934. https://doi.org/10.1016/i.cellsig.2011.01.021

11. Hahn C.G., Friedman E. Abnormalities in protein kinase $C$ signaling and the pathophysiology of bipolar disorder. Bipolar Disord. 1999, 1, 81-86. https://doi.org/10.1034/i.1399-5618.1999.010204.x

12. Iwamoto K., Kakiuchi C., Bundo M., Ikeda K., Kato T. Molecular characterization of bipolar disorder by comparing gene expression profiles of postmortem brains of major mental disorders. Mol Psychiatry. 2004, 9, 406-416. https://doi.org/10.1038/sj.mp.4001437

13. Wang H.Y., Friedman E. Enhanced protein kinase C activity and translocation in bipolar affective disorder brains. Biol Psychiatry. 1996, 40, 568-575. https://doi.org/10.1016/0006-3223(95)00611-7

14. Herrick S., Evers D.M., Lee J.Y., Udagawa N., Pak D.T. Postsynaptic PDLIM5/Enigma Homolog binds SPAR and causes dendritic spine shrinkage. Mol Cell Neurosci. 2010, 43, 188-200. https://doi.org/10.1016/i.mcn.2009.10.009

15. Horiuchi Y., Ishikawa M., Kaito N., lijima Y., Tanabe Y., Ishiguro H., et al. Experimental evidence for the involvement of PDLIM5 in mood disorders in hetero knockout mice. PLoS One. 2013, 8, e59320. https://doi.org/10.1371/journal.pone.0059320

16. Horiuchi Y., Arai M., Niizato K., Iritani S., Noguchi E., Ohtsuki T., et al. A polymorphism in the PDLIM5 gene associated with gene expression and schizophrenia. Biol Psychiatry. 2006, 59, 434-439. https://doi.org/10.1016/i.biopsych.2005.07.041

17. Liu Z., Liu W., Xiao Z., Wang G., Yin S., Zhu F., et al. A major single nucleotide polymorphism of the PDLIM5 gene associated with recurrent major depressive disorder. J Psychiatry Neurosci. 2008, 33, 43-46. https://www.ncbi.nlm.nih.gov/pubmed/18197271

18. Kato T., Iwayama Y., Kakiuchi C., Iwamoto K., Yamada K., Minabe Y., et al. Gene expression and association analyses of LIM (PDLIM5) in bipolar disorder and schizophrenia. Mol Psychiatry. 2005, 10, 1045-1055.

https://doi.org/10.1038/sj.mp.4001719 
19. Zain M.A., Roffeei S.N., Zainal N.Z., Kanagasundram S., Mohamed Z. Nonsynonymous polymorphisms of the PDLIM5 gene association with the occurrence of both bipolar disorder and schizophrenia. Psychiatr Genet. 2013, 23, $258-261$. https://doi.org/10.1097/YPG.0000000000000015

20. Zhao T., Liu Y., Wang P., Li S., Zhou D., Zhang D., et al. Positive association between the PDLIM5 gene and bipolar disorder in the Chinese Han population. J Psychiatry Neurosci. 2009, 34, 199-204.

https://www.ncbi.nlm.nih.gov/pubmed/19448850

21. Numata S., Ueno S., Iga J., Yamauchi K., Hongwei S., Hashimoto R., et al. Gene expression in the peripheral leukocytes and association analysis of PDLIM5 gene in schizophrenia. Neurosci Lett. 2007, 415, 28-33.

https://doi.org/10.1016/i.neulet.2007.01.018

22. Iga J., Ueno S., Yamauchi K., Numata S., Motoki I., Tayoshi S., et al. Gene expression and association analysis of LIM (PDLIM5) in major depression. Neurosci Lett. 2006, 400, 203-207. https://doi.org/10.1016/j.neulet.2006.02.044

23. Shi J., Badner J.A., Liu C. PDLIM5 and susceptibility to bipolar disorder: a family-based association study and metaanalysis. Psychiatr Genet. 2008, 18, 116-121. https://doi.org/10.1097/YPG.0b013e3282fa184b

24. Nichols D.E., Nichols C.D. Serotonin receptors. Chem Rev. 2008, 108, 1614-1641. https://doi.org/10.1021/cr0782240

25. Nichols C.D. Serotonin 5-HT(2A) Receptor function as a contributing factor to both neuropsychiatric and cardiovascular diseases. Cardiovasc Psychiatry Neurol. 2009, 2009, 475108. https://doi.org/10.1155/2009/475108

26. Amidfar M., Kim Y.K., Colic L., Arbabi M., Mobaraki G., Hassanzadeh G., Walter M. Increased levels of 5HT2A receptor mRNA expression in peripheral blood mononuclear cells of patients with major depression: correlations with severity and duration of illness. Nord J Psychiatry. 2017,71, 282-288, https://doi.org/10.1080/08039488.2016.1276624

27. Chee I.S., Lee S.W., Kim J.L., Wang S.K., Shin Y.O., Shin S.C., et al. 5-HT2A receptor gene promoter polymorphism 1438A/G and bipolar disorder. Psychiatr Genet. 2001, 11, 111-114. https://www.ncbi.nlm.nih.gov/pubmed/11702051

28. Gutierrez B., Bertranpetit J., Collier D., Arranz M.J., Valles V., Guillamat R., et al. Genetic variation of the 5-HT2A receptor gene and bipolar affective disorder. Hum Genet. 1997, 100, 582-584. https://www.ncbi.nlm.nih.gov/pubmed/9341875

29. Kishi T., Kitajima T., Tsunoka T., Ikeda M., Yamanouchi Y., Kinoshita Y., et al. Genetic association analysis of serotonin 2A receptor gene (HTR2A) with bipolar disorder and major depressive disorder in the Japanese population. Neurosci Res. 2009, 64, 231-234. https://doi.org/10.1016/i.neures.2009.03.003

30. Sherry S.T., Ward M.H., Kholodov M., Baker J., Phan L., Smigielski E.M., et al. dbSNP: the NCBI database of genetic variation. Nucleic Acids Res. 2001, 29, 308-311. https://www.ncbi.nlm.nih.gov/pubmed/11125122

31. Gordon D., Haynes C., Blumenfeld J., Finch S.J. PAWE-3D: visualizing power for association with error in case-control genetic studies of complex traits. Bioinformatics. 2005, 21, 3935-3937. https://doi.org/10.1093/bioinformatics/bti643

32. Shi Y.Y., He L. SHEsis, a powerful software platform for analyses of linkage disequilibrium, haplotype construction, and genetic association at polymorphism loci. Cell Research. 2006, 15, 97-98. https://doi.org/10.1038/sj.cr.7290272

33. Shen J., Li Z., Chen J., Song Z., Zhou Z., Shi Y. SHEsisPlus, a toolset for genetic studies on polyploid species. Sci. Rep. 2016, 6, 24095. https://doi.org/10.1038/srep24095

34. Li Z., Zhang Z., He Z., Tang W., Li T., Zeng Z., et al. A partition-ligation-combination-subdivision EM algorithm for haplotype inference with multiallelic markers. Cell Res. 2009, 19, 519-523. https://doi.org/10.1038/cr.2009.33

35. Lewis C.M. Genetic association studies: design, analysis and interpretation. Brief Bioinform. 2002, 3, $146-153$. https://www.ncbi.nlm.nih.gov/pubmed/12139434

36. Lewis C.M., Knight J. Introduction to genetic association studies. Cold Spring Harb Protoc. 2012, 3, $297-306$. https://doi.org/10.1101/pdb.top068163 